Research Article

\title{
The Prevalence and Determinants of Hand and Face Dermatitis during COVID-19 Pandemic: A Population-Based Survey
}

\author{
Mohammed Saud Alsaidan $\left(\mathbb{D},{ }^{1}\right.$ Aisha H. Abuyassin, ${ }^{2}$ Zahra H. Alsaeed, \\ Saqer H. Alshmmari, ${ }^{3}$ Tariq F. Bindaaj, ${ }^{3}$ and Alwa'ad A. Alhababi ${ }^{2}$ \\ ${ }^{1}$ Internal Medicine Department, College of Medicine, Prince Sattam Bin Abdulaziz University, Al-Kharj, Saudi Arabia \\ ${ }^{2}$ Dar Al Uloom University, Riyadh, Saudi Arabia \\ ${ }^{3}$ Prince Sattam Bin Abdulaziz University, Al-Kharj, Saudi Arabia
}

Correspondence should be addressed to Mohammed Saud Alsaidan; m.alsaidan@psau.edu.sa

Received 9 October 2020; Revised 18 November 2020; Accepted 24 November 2020; Published 9 December 2020

Academic Editor: E. Helen Kemp

Copyright (C) 2020 Mohammed Saud Alsaidan et al. This is an open access article distributed under the Creative Commons Attribution License, which permits unrestricted use, distribution, and reproduction in any medium, provided the original work is properly cited.

\begin{abstract}
Background. During the current COVID-19 pandemic, prevention is the key to limiting the spread of this disease. The frequent handwashing and use of sanitizers resulted in notable skin changes among some individuals. The aim of the study was to determine the prevalence and determinants of the new onset of dermatitis during the COVID-19 pandemic in a university population from Saudi Arabia. Methods. A cross-sectional study was conducted using a self-administered online questionnaire by sending an invitation link to students and employees of Prince Sattam Bin Abdulaziz University in June 2020. A chi-squared test was used to note differences regarding hand and face dermatitis. Results. Of the total 2356 participants, 34.8\% reported skin changes or symptoms over hands, and $15.3 \%$ reported skin changes on their face during this pandemic. $88.7 \%$ of the participants reported a change in handwashing habits during the COVID-19 pandemic, and $62.2 \%$ of participants were not using any hand sanitizers before COVID-19 but began using them during the pandemic. There were significantly higher percentage of skin conditions in females (on hands $(\mathrm{ScH}): 42.6 \%$ and face $(\mathrm{ScF}): 19.2 \%)$, individuals working in environments requiring frequent handwashing (ScH: $40.3 \%$ vs. ScF: $17.2 \%$ ), those working in facilities where they have to interact with people during the pandemic ( $\mathrm{ScH}: 41.1 \%$ vs. ScF: $18.7 \%$ ), those encountering COVID-19 patients ( $\mathrm{ScH}: 48.6 \%$ vs. ScF: $24.8 \%$ ), those exposed to chemicals (ScH: $48.6 \%$ vs. ScF: $24.8 \%$ ), and healthcare workers ( $\mathrm{ScH}$ : $51.3 \%$ vs. ScF: $24.3 \%)$. Conclusion. It was found that during the pandemic, skin changes were common among the general population as well as among healthcare workers. The frequency of handwashing and the use of alcohol-based sanitizers were contributing factors for dermatitis. Although hygiene is an extremely important preventive measure in this pandemic, maintaining skin integrity is also vital. Appropriate knowledge and good practice can prevent dermatitis in this pandemic, with regular hydration of the skin being a key factor.
\end{abstract}

\section{Introduction}

An ongoing pandemic caused by the coronavirus disease (COVID-19) has been reported globally, with millions of cases and hundreds of thousands of deaths to date [1]. These numbers are increasing daily around the world. The spread of this deadly infection is from person to person through close contact-either directly through the inhalation of small droplets produced as a result of sneezing, coughing, or talking by infected persons or indirectly through contact with contaminated surfaces [2]. Currently, there is no effective antiviral treatment or vaccine available against this virus. The only way to prevent and reduce the rate of this infection is by using surface disinfectants, regularly washing hands, using hand sanitizers, covering the mouth and face with face masks, and using personal protective equipment (PPE) [3].

Evidence has indicated that countries that strictly followed guidelines of complete lockdown, physical distancing, and 
prevention strategies reported a reduced burden and fewer cases of COVID-19. Hand hygiene comes first in prevention strategies and for the reduction of hospital-acquired infection [4]. Hand hygiene with soaps and water or by using an alcoholbased sanitizer is the widely used method that is cheap, effective, and simple against COVID-19 [5]. The WHO emphasizes hand hygiene at the right times in a sufficient way in order to prevent the spread by $50 \%[3,6]$.

Alcohol is commonly used in sanitizers as it possesses disinfectant and biocidal properties. These properties vary depending on the strength, type, and antiviral activity of the alcohol being used in sanitizers [7]. In 2017, the WHO developed two hand rub formulations for the prevention of enveloped viruses [8]. Antiviral and antibacterial activity of sanitizers increases with higher concentrations of alcohol mixed with other organic/inorganic acids $[9,10]$. The efficacy of sanitizers in preventing the spread of many pathogens has been well documented [11]. However, precautions must be taken when frequently using sanitizers, as the excessive use of these agents may cause side effects. For instance, oils secreted by the sebaceous glands of the skin have inherent antiviral properties [12]. The continual use of alcohol-based hand sanitizers washes away these oils, leaving the skin dehydrated, which in turn results in fissures and erosion, allowing easy access to pathogens and increasing the risk of microbial infection [3]. The use of alcoholbased hand sanitizers markedly increased following the recommendations suggested by the Centers for Disease Control and Prevention (CDC) and WHO to fight against COVID-19 [13]. Consequently, this not only led to panic buying and overuse of sanitizers but also increased the supply of substandard products in the markets [14]. These products have limited antiviral activity, leading to the emergence and re-emergence of some microorganisms and dermatological problems [15].

The excessive use of soaps (bars or liquid) and detergents is also harmful. Frequent and prolonged handwashing causes a disruption of the lipid barrier (epidermal), resulting in increased skin sensitivity to any physical and chemical agents [16]. Although soaps and detergents are weak irritants, overzealous use of them causes cumulative irritant contact dermatitis and redness of the skin, mainly over the back of the hand and the webs of the fingers [16]. Other dermatological problems include contact cutaneous xerosis, flaking, allergic or irritant contact, dermatitis, and eczema $[17,18]$. Some countries reported an increased incidence of skin problems during this pandemic [19-22]. In light of the aforementioned concerns and the increased incidence of hand eczema reported around the world, this study aims to identify the prevalence and associated factors of dermatitis during the COVID-19 pandemic in Saudi Arabia.

\section{Materials and Methods}

2.1. Study Design. A cross-sectional study was conducted in June 2020.

2.2. Study Setting and Study Population. A total of 2356 individuals participated in the study. An invitation link was sent to students and employees of Prince Sattam Bin
Abdulaziz University, Al-Kharj, Saudi Arabia. Participants who accepted the invitation filled the online questionnaire. Individuals from both the general population and healthcare workers were included in the study. All the data were collected anonymously through SurveyHero online. The study design obtained the required ethical approvals of the ethics committee of the Prince Sattam Bin Abdulaziz University (PSAU/COM/RC/IRB/p/80). The consent to participate was a part of the questionnaire.

2.3. Data Collection Tools. The data were collected using the self-administered questionnaire.The questionnaire was administered in both Arabic and English versions and was translated back and forth by two bilingual translators. The questionnaire was developed after an in-depth literature review and was reviewed by two independent dermatologists. A pilot study of 30 participants was conducted, and the questionnaire was adjusted accordingly.

2.4. Statistical Analysis. A descriptive analysis was conducted. Mean/median and standard deviation (SD)/ interquartile range (range) were reported for continuous variables, while frequency and percentages were calculated for categorical variables. The Shapiro-Wilk (S-W) tests were conducted for the variable age and showed a $p$ value of 0.01 , indicating that age variable was not normally distributed in our sample population. A chi-squared test was conducted to observe differences with respect to hand and face dermatitis. A $p$ value of 0.05 or less was considered significant. Data are reported in tabular and graphical form. SPSS version 25 was used to conduct the analysis.

\section{Results}

Data from 2356 individuals were collected. Of the total number of participants, $55.9 \%$ were females, $76.4 \%$ were single, and the majority $(93.0 \%)$ were Saudi nationals. The median age of participants was 21.00 (IQR: 18-26). 27.8\% were employed or self-employed, and $56.1 \%$ were students. $64.5 \%$ of respondents had a monthly family income that was less than 10000 Saudi Riyals (Table 1).

When asked about their handwashing practices, $42.6 \%$ of the employees reported that they work in settings that require frequent handwashing, and $38.6 \%$ reported that they work in settings that necessitate encounters with customers. Additionally, $14.6 \%$ reported working in settings that require the use of irritating materials like chemicals, soaps, or other detergents. Only $4.5 \%$ of participants were working in settings in which there was direct contact with COVID-19 patients, and $6.9 \%$ were healthcare workers (Table 1). Moreover, $12 \%$ of participants had eczema, $9 \%$ had asthma, $2.3 \%$ had urticaria, $7.4 \%$ had rhinitis, $0.7 \%$ had rosacea, $19.7 \%$ had acne, and $5.8 \%$ reported having other skin conditions.

More than two-thirds $(88.7 \%)$ of the participants reported that their handwashing habits changed during the COVID-19 pandemic. $42.2 \%$ reported washing their hands 3.5 times per day before the COVID-19 pandemic, but 
TABLE 1: Sociodemographics and hand hygiene practices during the COVID-19 pandemic, and the prevalence of skin conditions ( $n=2354)$.

\begin{tabular}{|c|c|c|c|c|c|}
\hline \multirow[t]{2}{*}{ Variables } & \multirow[t]{2}{*}{ Total $n=2354(\%)$} & \multicolumn{2}{|c|}{$\begin{array}{l}\text { Skin conditions on hand } \\
\qquad(n=821)\end{array}$} & \multicolumn{2}{|c|}{$\begin{array}{l}\text { Skin conditions on face } \\
(n=360)\end{array}$} \\
\hline & & Yes $(\%)$ & $p$ value & Yes $(\%)$ & $p$ value \\
\hline \multicolumn{6}{|l|}{ Gender } \\
\hline Male & $1038(44.1)$ & $260(25.0)$ & $<0.001$ & $107(10.3)$ & \multirow{2}{*}{$<0.001$} \\
\hline Female & $1318(55.9)$ & $561(42.6)$ & - & $235(19.2)$ & \\
\hline \multicolumn{6}{|l|}{ Marital Status } \\
\hline Single & $1842(78.2)$ & $604(32.8)$ & $<0.001$ & $272(14.8)$ & \multirow{2}{*}{0.19} \\
\hline Married & $514(21.8)$ & $217(42.2)$ & - & $88(17.1)$ & \\
\hline \multicolumn{6}{|l|}{ Employment } \\
\hline Employed & $656(27.8)$ & $281(42.8)$ & $<0.001$ & $123(18.8)$ & \multirow{2}{*}{0.004} \\
\hline Housewife/Student & $1700(72.2)$ & $540(31.8)$ & - & $237(13.9)$ & \\
\hline \multicolumn{6}{|l|}{ Healthcare worker } \\
\hline Yes & $163(6.9)$ & $95(58.3)$ & $<0.001$ & $54(33.1)$ & \multirow{2}{*}{$<0.001$} \\
\hline No & $2193(93.1)$ & $726(33.1)$ & - & $306(14.0)$ & \\
\hline \multicolumn{6}{|l|}{ Handwashing* } \\
\hline Yes & $1003(42.6)$ & $404(40.3)$ & $<0.001$ & $173(17.2)$ & \multirow{2}{*}{0.022} \\
\hline No & $1353(57.4)$ & $417(30.8)$ & - & $187(13.8)$ & \\
\hline \multicolumn{6}{|c|}{ Encounter with people having COVID-19* } \\
\hline Yes & $910(38.6)$ & $374(41.1)$ & $<0.001$ & $170(18.7)$ & \multirow{2}{*}{$<0.001$} \\
\hline No & $1446(61.4)$ & $447(30.9)$ & - & $190(13.1)$ & \\
\hline \multicolumn{6}{|c|}{ Encounter with COVID-19 patients* } \\
\hline Yes & $105(4.5)$ & $51(48.6)$ & 0.003 & $26(24.8)$ & \multirow{2}{*}{0.006} \\
\hline No & $2251(95.5)$ & $770(34.2)$ & - & $334(14.8)$ & \\
\hline \multicolumn{6}{|l|}{ Use of chemicals* } \\
\hline Yes & $345(14.6)$ & $177(51.3)$ & $<0.001$ & $84(24.3)$ & \multirow{2}{*}{$<0.001$} \\
\hline No & $2011(85.4)$ & $644(32.0)$ & - & $276(13.7)$ & \\
\hline
\end{tabular}

during the pandemic, the frequency of handwashing increased drastically $6-20$ times for $70.4 \%$ of individuals. Nearly two-thirds (68.6\%) reported washing their hands for less than 1 minute every time, while $27.4 \%$ reported washing it for 1-2 minutes. $82.6 \%$ reported using lukewarm water when washing their hands. Nearly half reported using antiseptic soaps for washing their hands, $53.4 \%$ reported using regular soaps, $52.0 \%$ reported using perfumed soaps, and $85 \%$ reported using liquid soaps (Table 2).

Regarding the usage of hand sanitizers, $87.6 \%$ reported that their habit of using hand sanitizers has changed during the pandemic. $62.2 \%$ were not using any hand sanitizers before COVID-19, but during the pandemic, $53.7 \%$ were using them 3-10 times per day, and $23.5 \%$ were using perfumed sanitizers. Of the total number of participants, $77 \%$ preferred handwashing to using hand sanitizers. $67.6 \%$ reported using hand sanitizers after coming into contact with any person or surface, while $21 \%$ were using it without getting in contact with anyone. In addition, 62\% used it every time they entered their homes after being outside, and 48.5\% reported using it before and after eating (Table 2).

Furthermore, $70.1 \%$ reported that they believed that excessive handwashing or the excessive use of sanitizers could cause skin problems. In regard to glove usage, $42.1 \%$ were not using any gloves, with $10.8 \%$ of participants being reported not using gloves because of skin problems. Out of those who did wear gloves, $35.6 \%$ used 1-2 pairs per day. With respect to face masks, $75.5 \%$ reported using surgical/ medical face masks; $54.2 \%$ of the participants used the masks for half an hour to 2 hours; and $66.8 \%$ reported changing it 1-2 times per day.

Of the total number of respondents, $34.8 \%$ (821 individuals) reported skin changes or symptoms during COVID-19, of whom $83.2 \%$ reported skin dryness, $54.2 \%$ reported changes in the texture, $45.4 \%$ reported scaling, $39.6 \%$ reported itchiness, $28.4 \%$ reported changes in skin color, $28.1 \%$ reported redness, and $17.4 \%$ reported pain/ burning, while $7.6 \%$ reported skin ulcers (Figure 1 ). In order to alleviate these symptoms, $77.9 \%$ began to use moisturizers, $19.7 \%$ reported limiting handwashing and sanitizing, $7.2 \%$ consulted a doctor, and 5.6\% used topical steroids. Only $29.8 \%$ reported improvements in skin changes.

$15.3 \%$ (360 individuals) reported skin changes on their faces during the pandemic. $4.9 \%$ of those with skin changes believed that they were due to the usage of face masks. However, the symptoms were mild for most $(87.2 \%)$ of the patients. One hundred sixty-three of the participants were healthcare workers, and among them, 78.5\% were working in hospitals (Figure 2).

There is a significantly higher prevalence of figures of skin conditions on patients' hands and face in the following demographics: females (skin condition on hands $(\mathrm{ScH})$ : $42.6 \%$ and skin condition on face $(\mathrm{ScF}): 19.2 \%)$, married individuals ( $\mathrm{ScH}: 42.2 \%$ and $\mathrm{ScF}: 17.3 \%$ ), those who were employed ( $\mathrm{ScH}$ : $42.8 \%$ and $\mathrm{ScF}: 18.8 \%$ ), individuals working in environments that require handwashing several times (ScH: $40.3 \%$ vs. ScF: $17.2 \%$ ), those who were working in facilities where they have to interact with people during the 
TABLE 2: Factors leading to skin conditions on hands during the COVID-19 pandemic $(n=821)$.

\begin{tabular}{|c|c|c|c|}
\hline \multirow[t]{2}{*}{ Variables } & \multirow[t]{2}{*}{ Total $n=2354(\%)$} & \multicolumn{2}{|c|}{$\begin{array}{l}\text { Skin conditions on } \\
\text { hand }(n=821)\end{array}$} \\
\hline & & Yes $(\%)$ & $p$ value \\
\hline \multicolumn{4}{|c|}{ Handwashing habit changed } \\
\hline Yes & $2090(88.7)$ & $46(17.3)$ & \multirow{2}{*}{$<0.001$} \\
\hline No & $266(11.3)$ & $775(37.1)$ & \\
\hline \multicolumn{4}{|l|}{ Handwashing } \\
\hline$\leq 10$ times & $1338(56.8)$ & $382(38.6)$ & \multirow{2}{*}{$<0.001$} \\
\hline$>10$ times & $1018(43.2)$ & $439(43.1)$ & \\
\hline \multicolumn{4}{|l|}{ Washing time } \\
\hline$<1 \min ^{\circ}$ & $1617(68.6)$ & $535(33.1)$ & \multirow{3}{*}{0.030} \\
\hline $1-2 \mathrm{~min}$ & $646(27.4)$ & $250(38.7)$ & \\
\hline$>2 \min$ & $93(3.9)$ & $36(38.7)$ & \\
\hline \multicolumn{4}{|c|}{ Handwashing water } \\
\hline Luke warm & $1945(82.6)$ & $693(35.6)$ & \multirow{3}{*}{0.003} \\
\hline Cold & $231(9.8)$ & $58(25.1)$ & \\
\hline Hot & $180(7.6)$ & $70(38.9)$ & \\
\hline \multicolumn{4}{|l|}{ Type of soap } \\
\hline Regular & $1257(53.4)$ & $428(34.0)$ & \multirow{2}{*}{0.385} \\
\hline Antiseptic & $1099(46.6)$ & $393(35.8)$ & \\
\hline \multicolumn{4}{|l|}{ Perfumed soap } \\
\hline Yes & $1226(52.0)$ & $431(35.2)$ & \multirow{2}{*}{0.744} \\
\hline No & $1130(48.0)$ & $390(34.5)$ & \\
\hline \multicolumn{4}{|l|}{ Type of soap } \\
\hline Solid & $353(15.0)$ & $116(32.9)$ & \multirow{2}{*}{0.396} \\
\hline Liquid & $2003(85.0)$ & $705(35.2)$ & \\
\hline \multicolumn{4}{|c|}{ Changed in hand sanitizer habit } \\
\hline Yes & $2064(87.6)$ & $750(36.3)$ & \multirow{2}{*}{$<0.001$} \\
\hline No & $292(12.4)$ & $71(24.3)$ & \\
\hline Hand sanitizer pe & day & & \\
\hline$\leq 10$ times & $1864(79.1)$ & $628(33.7)$ & \\
\hline$>10$ times & $492(20.9)$ & $193(39.2)$ & 0.022 \\
\hline Alcohol concentra & & & \\
\hline$<60 \%$ & $191(8.1)$ & $77(40.3)$ & \\
\hline$>60 \%$ & $503(21.3)$ & $232(46.1)$ & $<0.001$ \\
\hline Do not know & $1662(70.5)$ & $512(30.8)$ & \\
\hline Perfumed sanitize & & & \\
\hline Yes & $554(23.5)$ & $187(33.8)$ & \\
\hline No & $1802(76.5)$ & $634(35.2)$ & 0.537 \\
\hline Sanitizing when $g$ & ing in contact & & \\
\hline Yes & $1593(67.6)$ & $602(37.8)$ & \\
\hline No & $763(32.4)$ & $219(28.7)$ & $<0.001$ \\
\hline Every one or two & urs & & \\
\hline Yes & $495(21.0)$ & $202(40.8)$ & \\
\hline No & $1861(79.0)$ & $619(33.3)$ & 0.002 \\
\hline After coming from & utside & & \\
\hline Yes & $1473(62.5)$ & $536(36.4)$ & 0043 \\
\hline No & $883(37.5)$ & $285(32.3)$ & 0.043 \\
\hline Before and after e & ing & & \\
\hline Yes & $1143(48.5)$ & $442(38.7)$ & $<0001$ \\
\hline No & $1213(51.5)$ & $379(31.2)$ & $<0.001$ \\
\hline Hand gloves/day & & & \\
\hline Do not wear & $1093(46.4)$ & $351(32.1)$ & \\
\hline$>1$ pair & $1263(53.6)$ & $470(37.2)$ & 0.010 \\
\hline Stopped using glov & & & \\
\hline Yes & $254(10.8)$ & $149(58.7)$ & \\
\hline No/do not know & $2102(89.2)$ & $672(32.0)$ & 1 \\
\hline
\end{tabular}

TABLE 2: Continued.

\begin{tabular}{lccc}
\hline Variables & Total $n=2354(\%)$ & \multicolumn{2}{c}{$\begin{array}{c}\text { Skin conditions on } \\
\text { hand }(n=821)\end{array}$} \\
& & Yes $(\%)$ & $p$ value \\
\hline $\begin{array}{l}\text { Had hand eczema } \\
\text { Yes }\end{array}$ & $355(15.1)$ & $198(55.8)$ & $<0.001$ \\
No & $2001(84.9)$ & $623(31.1)$ & \\
\hline $\begin{array}{l}\text { Household work } \\
\leq 2 \text { hours/week }\end{array}$ & $1502(63.8)$ & $499(33.2)$ & 0.028 \\
$>2$ hours & $854(36.2)$ & $322(37.7)$ & \\
\hline
\end{tabular}

pandemic (ScH:41.1\% vs. ScF: $18.7 \%$ ), those who were encountering COVID-19 patients (ScH: $48.6 \%$ vs. ScF: $24.8 \%$ ), those working in facilities where they have to work with chemicals (ScH: $48.6 \%$ vs. ScF: $24.8 \%$ ), and healthcare workers (ScH: $51.3 \%$ vs. ScF: $24.3 \%$ ) [Table 1].

Skin conditions on hands were significantly more common among specific groups, including individuals who did not change their handwashing habits during the pandemic (37.1\%), those who washed their hands several times per day (43.1\%), those who washed more than 2 minutes (38.7\%), or those who washed hands with cold water (38.9\%). Skin conditions were also reported to be significantly more common among individuals who altered their usage of hand sanitizers during the pandemic (36.3\%), those who used sanitizers several times per day (39.2\%), those who used sanitizers with a greater alcohol concentration (46.1\%), those who sanitized every one to two hours $(40.8 \%)$, those who sanitized after coming from outside (36.4\%), and those who sanitized before and after eating (38.7\%) (Table 2).

Similarly, individuals who were using hand gloves (37.2\%), who previously had eczema (55.8\%), and who reported doing household work for more than 2 hours (37.7\%) had significantly more skin conditions (58.3\%). However, while skin conditions on the face were related to wearing facemasks, the duration of wearing facemasks and the number of facemasks changed per day did not affect skin conditions (Table 3 ).

\section{Discussion}

This population-based study was conducted to determine the prevalence of hand dermatitis in Saudi Arabia during the ongoing COVID-19 pandemic. In the study, most participants were Saudi nationals, young, females, single, and employed, while $7 \%$ were healthcare workers. Around twofifths of the participants practiced frequent handwashing. The majority of participants reported that their handwashing habits changed, with their frequency of handwashing increasing from three times a day to 20 times a day during this pandemic. Two-third of the participants washed their hands for $<1 \mathrm{~min}$, while one-third washed them for 1-2 min every time. Half of the participants used soaps and water, while $77 \%$ used hand sanitizers. $35 \%$ of participants reported skin changes, with skin dryness being frequently presented. As a result of these changed washing and sanitizing habits among 


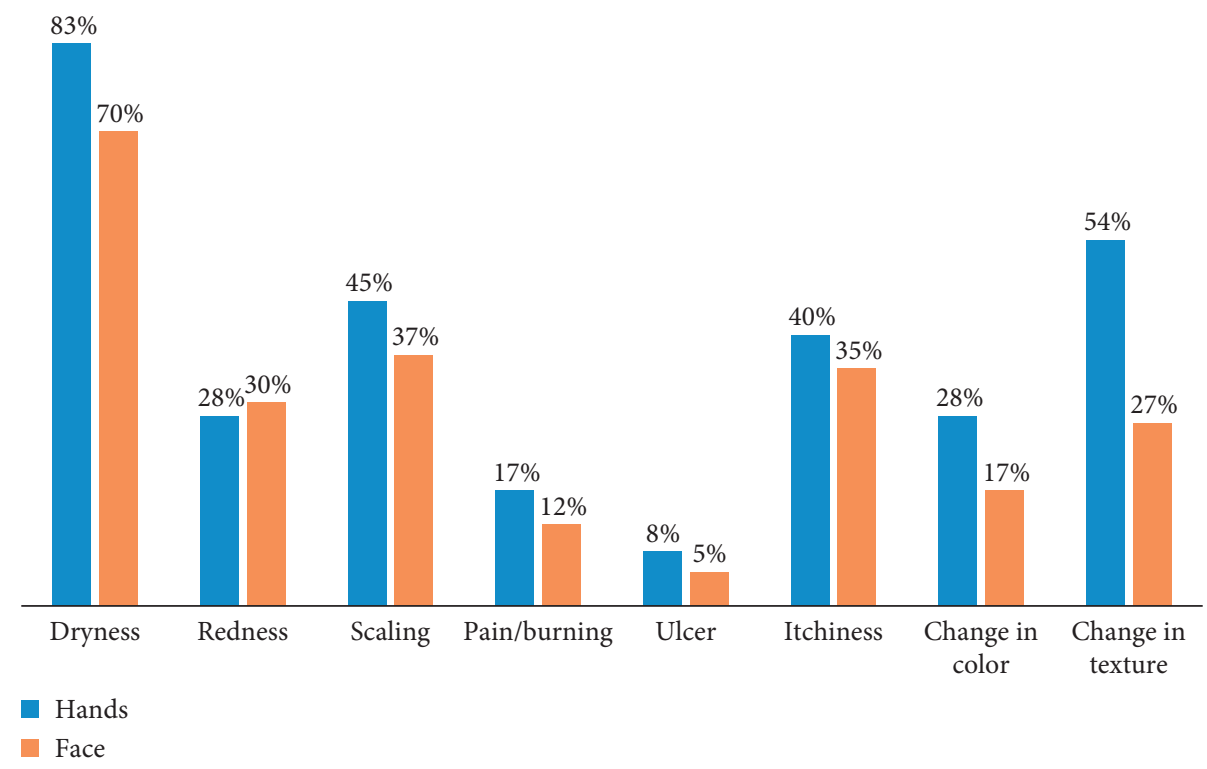

Figure 1: Skin conditions on hands and the face during the pandemic $(n=2356)$.

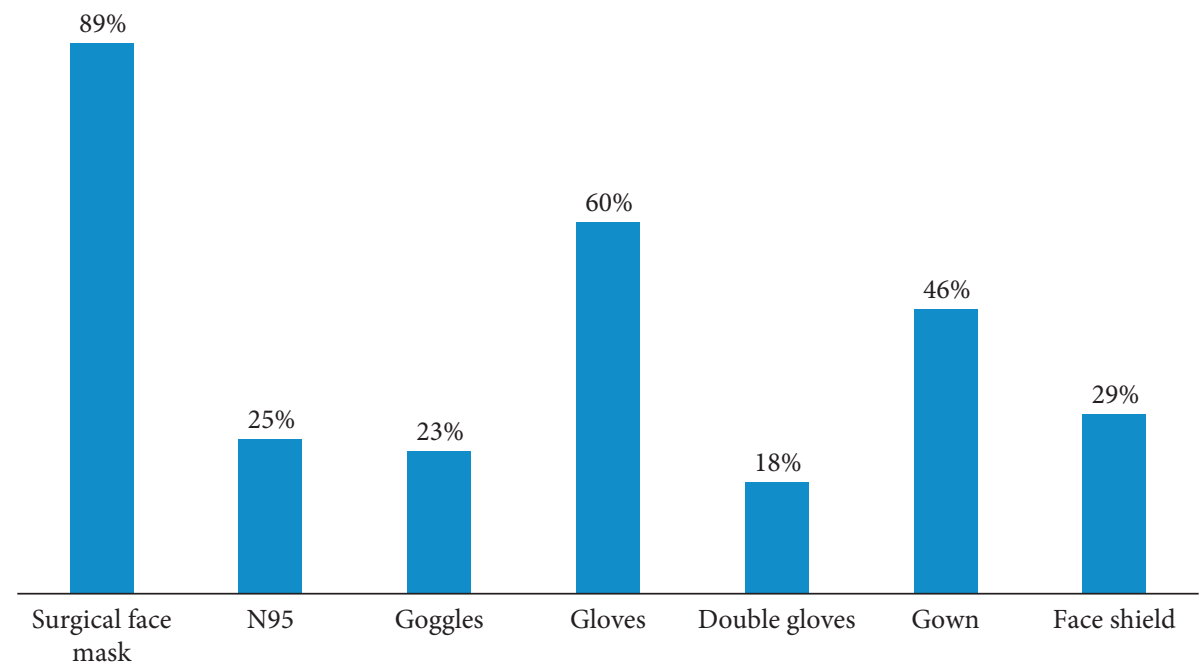

FIGURE 2: PPE used by healthcare workers during the pandemic $(n=163)$.

the Saudi population, $34.8 \%$ reported skin changes over hands and $15.1 \%$ over the face.

Dermatitis is an inflammatory response of the skin caused by allergens, irritant substances, or both [23]. As seen in this study, frequent handwashing altered the skin texture, with changes ranging from dryness to dermatitis. In this study, more than one-third reported skin changes, mostly skin dryness and altered skin texture, with some cases of redness, scaling, pain, itching, or even ulcers. A review by Cristina Beiu highlighted these potential adverse effects that can be caused by immoderate handwashing [17]. A study conducted in India found 16 new cases of hand eczema in the general population due to excessive hand hygiene practices [19]. Similarly, a Chinese study reported a $74.5 \%$ prevalence of hand eczema in healthcare providers [20], while another study reported a $90.2 \%$ incidence of eczema among German healthcare workers [21]. Moreover, a study conducted in Milan, Italy, reported an increased frequency of hand eczema [22].

It is also known that, in addition to sanitizers, the constant and prolonged use of soap water in humid environments causes the disruption of the skin's outer layer, which in turn increases the skin's permeability to various agents [24]. Furthermore, stress, atmospheric aspects, quarantine, and lockdowns increase the prevalence and severity of many disease including atopic dermatitis [25]. Wet work (working with one's hands in a wet environment for $>2$ hours a day, or washing hands $>20$ times a day) and gloves occlusion (using waterproof gloves for $>2$ hours) causes skin barrier impairment, which, when combined with exposure to soaps or sanitizers, can trigger irritant contact dermatitis [26]. This can also be seen in individuals who wear gloves for a prolonged time. Healthcare workers working in COVID-19 wards have to wear PPE for several hours and, 
TABLE 3: Factors leading to skin conditions on the face during the COVID-19 pandemic $(n=821)$.

\begin{tabular}{|c|c|c|c|}
\hline \multirow[t]{2}{*}{ Variables } & \multirow[t]{2}{*}{ Total $n=2354(\%)$} & \multicolumn{2}{|c|}{$\begin{array}{l}\text { Skin conditions on face } \\
\qquad(n=360)\end{array}$} \\
\hline & & Yes (\%) & $p$ value \\
\hline \multicolumn{4}{|l|}{ Face mask } \\
\hline Surgical & $1779(75.5)$ & $291(16.4)$ & \multirow{3}{*}{0.019} \\
\hline Others & $430(18.3)$ & $47(10.9)$ & \\
\hline Do not use & $147(6.2)$ & $22(15.0)$ & \\
\hline \multicolumn{4}{|c|}{ Face mask hours } \\
\hline$</=2$ hours & $1277(54.2)$ & $187(14.6)$ & \multirow{3}{*}{0.645} \\
\hline$>2$ hours & $713(30.3)$ & $114(16.0)$ & \\
\hline Do not use & $366(15.5)$ & $59(16.1)$ & \\
\hline \multicolumn{4}{|c|}{ Facemask change } \\
\hline $1-2$ times & $1573(66.8)$ & $237(15.1)$ & \multirow{3}{*}{0.920} \\
\hline$>2$ times & $364(15.4)$ & $57(15.7)$ & \\
\hline Do not use & $419(17.8)$ & $66(5.8)$ & \\
\hline
\end{tabular}

hence, are susceptible to adverse skin reactions [27]. Other published studies have shown that the use of gloves can lead to the development of contact dermatitis $[6,28]$. Likewise, another study showed that the odds of developing dermatitis increased three times with the use of $>5$ pairs of gloves [29]. It was also found that dermatitis was significantly prevalent in $37 \%$ of individuals who used gloves ( $>1$ pair per day).

It was found that most of the skin changes (46\%) were significantly present in participants who used sanitizers with an alcohol concentration of more than $60 \%$. According to the WHO's recommendation, when one's hands are not visibly dirty or when soaps and water are not available, alcohol-based hand rubs (ABHRs) with an alcohol concentration of $>60 \%$ are the most appropriate alternative [5]. Similarly, in another study, all patients who came for dermatologic consultation because of skin damage had been washing their hands $>10$ times a day and had been using alcohol-based gel [22]. Since coronavirus is enveloped in a lipid bilayer, an alcohol-based sanitizer is undoubtedly effective. However, its excessive use can create multiple problems if not controlled in a timely manner. The major problems are the existence of substandard products in the markets as well as the emergence of alcohol tolerance, antimicrobial resistance (AMR), opportunistic infection, and product toxicity [30-32].

Dermatitis is significantly more prevalent among the healthcare workers and individuals who have contact with COVID-19 patients in this study, as they follow strict hygiene practices and wear PPE for a longer period of time. Similarly, a study reported a $97 \%$ prevalence of general skin damage in healthcare workers caused by excessive hygienic measures [20]. Another study reported a $90.4 \%$ prevalence of acute hand dermatitis and a $14.9 \%$ prevalence of hand eczema among healthcare workers [21]. Recent published studies also reported high prevalence figures of eczema in healthcare workers $[6,33]$.

In this study, some of the participants also faced skin changes on their faces due to the excessive use of face masks and PPE. A recent study reported that $97 \%$ of the skin damage was due to enhanced protective measures, and these include $83 \%$ of the nasal bridge lesion [34]. Other studies reported similar findings in addition to reporting other dermatologic side effects like pressure injury, urticaria, dryness of the skin, allergic contact dermatitis, and aggravation of underlying dermatosis. In all of these conditions, occlusion and friction were the main contributing factors [35-38]. These results were more commonly found among healthcare workers, who wear protective gear for prolonged times [20].

There are some limitations to the study. Firstly, only an online survey was conducted, with no dermatologist confirming the diagnosis. Secondly, the study is cross-sectional, so although the majority of the participants reported skin changes after the excessive use of hand hygiene, a temporal relationship and causality cannot be concluded. This can be better evaluated through prospective studies. Furthermore, all the symptoms were self-reported; therefore, the chance of recall bias cannot be excluded. However, the major strength of the study is that it covers both the general population and healthcare workers, in addition to using a relatively large sample size. Although the study was conducted on a large sample size, it only included students and employees of Prince Sattam Bin Abdulaziz University; therefore, the results may not be applicable to the general population and further studies are needed.

\section{Conclusion}

In conclusion, this study found that during this pandemic, skin changes were common among the general population as well as healthcare workers. This is mainly due to excessive handwashing and the use of alcohol-based sanitizers. Changes in washing habits, the frequency ( $>10$ times) and duration ( $>2 \mathrm{~min}$ ) of handwashing, and sanitizer alcohol concentration $(>60 \%)$ are also contributing factors. Although hygiene is a crucial preventive measure in this pandemic, maintaining skin integrity is also of the utmost importance. It is also known from previous studies that the disruption of the skin barrier can provide a route of entry for the coronavirus as the angiotensinconverting enzyme (ACE-2) - the cell receptor for COVID19-is present in hair follicles, the epidermis, and the blood vessels of the skin [19]. Further, only $7.3 \%$ of those who developed skin changes looked for medical assessment during this pandemic. Proper awareness and sufficient practice can prevent skin changes during this pandemic, especially because dermatitis is easily preventable and manageable, with regular hydration of the skin. Also, educating the public about using over-the-counter medications-if needed-could also be a key factor to prevent further complications for those who cannot visit a physician during a pandemic.

\section{Data Availability}

The data that support the study can be obtained from the corresponding author upon request.

\section{Consent}

No written consent has been obtained from the patients as there is no patient identifiable data included. 


\section{Conflicts of Interest}

The authors declare that they have no conflicts of interest.

\section{Acknowledgments}

This study was supported by the Deanship of Scientific Research at Prince Sattam Bin Abdulaziz University, Alkharj, Saudi Arabia.

\section{References}

[1] “Coronavirus disease (COVID-19) situation report-206," 2020, https://www.who.int/docs/default-source/coronaviruse/situationreports/20200813-covid-19-sitrep-206.pdf?sfvrsn=bf38f66b_6.

[2] Y. Bai, L. Yao, T. Wei, F. Tian, D.-Y. Jin, and L. Chen, "Presumed asymptomatic carrier transmission of COVID19," JAMA, vol. 323, no. 14, pp. 1406-1407, 2020.

[3] D. Pradhan, P. Biswasroy, P. Kumar Naik, G. Ghosh, and G. Rath, "A review of current interventions for COVID-19 prevention," Archives of Medical Research, vol. 51, no. 5, pp. 363-374, 2020.

[4] E. E. Sickbert-Bennett, L. M. DiBiase, T. M. Willis, E. S. Wolak, D. J. Weber, and W. A. Rutala, "Reduction of healthcare-associated infections by exceeding high compliance with hand hygiene practices," Emerg Infect Dis, vol. 22, pp. 1628-1630, 2016.

[5] WHO, Infection Prevention and Control during Health Care when Novel Coronavirus ( $\mathrm{nCoV}$ ) Infection is Suspected. Interim Guidance, WHO, Geneva, Switzerland, 2020, https:// apps.who.int/iris/rest/bitstreams/1266296/retrieve\%202020.

[6] N. Lotfinejad, A. Peters, and D. Pittet, "Hand hygiene and the novel coronavirus pandemic: the role of healthcare workers," Journal of Hospital Infection, vol. 105, no. 4, pp. 776-777, 2020.

[7] J. Jing, T. Yi, J. C. B. Rajendran, J. McCarthy, N. Tharmalingam, and T. Madheswaran, "Hand sanitizers: a review on formulation aspects, adverse effects, and regulations," International Journal of Environmental Research and Public Health, vol. 17, p. 3326, 2020.

[8] A. Siddharta, S. Pfaender, N. J. Vielle, R. Dijkman, M. Friesland, and B. Becker, "Virucidal activity of World Health Organization-recommended formulations against enveloped viruses, including zika, ebola, and emerging coronaviruses," The Journal of Infectious Diseases, vol. 215, no. 6, pp. 902-906, 2017.

[9] K. M. Cottingim, H. Verma, P. E. Urriola, F. Sampedro, G. C. Shurson, and S. M. Goyal, "Feed additives decrease survival of delta coronavirus in nursery pig diets," Porcine Health Management, vol. 3, no. 1, p. 5, 2017.

[10] G. Ionidis, J. Hübscher, T. Jack, B. Becker, B. Bischoff, and D. Todt, "Development and virucidal activity of a novel alcohol-based hand disinfectant supplemented with urea and citric acid," BMC Infectious Diseases, vol. 16, no. 1, p. 77, 2016.

[11] J. de Witt Huberts, K. Greenland, W.-P. Schmidt, and V. Curtis, "Exploring the potential of antimicrobial hand hygiene products in reducing the infectious burden in lowincome countries: an integrative review," American Journal of Infection Control, vol. 44, no. 7, pp. 764-771, 2016.

[12] P. W. Wertz, "Lipids and the permeability and antimicrobial barriers of the skin," Journal of Lipids, vol. 2018, Article ID 5954034, 2018.

[13] Centers for Disease Control and Prevention USA, Hand Hygiene Recommendations Guidance for Healthcare Providers about Hand Hygiene and COVID-19, Centers for
Disease Control and Prevention, Atlanta, GA, USA, 2020, https://www.cdc.gov/coronavirus/2019-ncov/hcp/handhygiene.html.

[14] A. Berardi, D. R. Perinelli, H. A. Merchant, L. Bisharat, I. A. Basheti, and G. Bonacucina, "Hand sanitisers amid COVID-19: a critical review of alcohol-based products on the market and formulation approaches to respond to increasing demand," International Journal of Pharmaceutics, vol. 2020, Article ID 119431, 2020.

[15] A. Emami, F. Javanmardi, A. Keshavarzi, and N. Pirbonyeh, "Hidden threat lurking behind the alcohol sanitizers in COVID-19 outbreak," Dermatology and Therapy, vol. 33, no. 4, p. e13627, 2020.

[16] R. Jindal and D. Pandhi, "Hand hygiene practices and risk and prevention of hand eczema during the COVID-19 pandemic," Indian Dermatol Online Journal, vol. 11, no. 4, pp. 540-543, 2020.

[17] C. Beiu, M. M. Mihai, L. Popa, L. Cima, and M. Popescu, "Frequent hand washing for COVID-19 prevention can cause hand dermatitis: management tips," Cureus, vol. 12, 2020.

[18] R. Darlenski and N. Tsankov, "COVID-19 pandemic and the skin: what should dermatologists know?" Clinics in Dermatology, 2020, In press.

[19] M. Singh, M. Pawar, A. Bothra, and N. Choudhary, "Overzealous hand hygiene during the COVID 19 pandemic causing an increased incidence of hand eczema among general population," Journal of the American Academy of Dermatology, vol. 83, no. 1, pp. e37-e41, 2020.

[20] J. Lan, Z. Song, X. Miao, H. Li, Y. Li, and L. Dong, "Skin damage among health care workers managing coronavirus disease-2019," Journal of the American Academy of Dermatology, vol. 82, no. 5, pp. 1215-1216, 2020.

[21] A. Guertler, N. Möllhoff, T. Schenck, C. Hagen, B. Kendziora, and R. Giunta, "Onset of occupational hand eczema among healthcare workers during the SARS-CoV-2 pandemic -comparing a single surgical site with a COVID-19 intensive care unit," Contact Dermatitis, vol. 83, no. 2, pp. 108-114, 2020.

[22] S. Giacalone, P. Bortoluzzi, and G. Nazzaro, "The fear of COVID-19 infection is the main cause of the new diagnoses of hand eczema: report from the frontline in Milan," Dermatologic Therapy, vol. 33, no. 4, p. e13630, 2020.

[23] J. D. Johansen, K. Aalto-Korte, T. Agner, K. E. Andersen, A. Bircher, and M. Bruze, "European Society of contact dermatitis guideline for diagnostic patch testing," Recommendations on Best Practice, vol. 73, no. 4, pp. 195-221, 2015.

[24] C. Patruno, G. Fabbrocini, L. Stingeni, and M. Napolitano, "The role of occupational dermatology in the COVID-19 outbreak," Contact Dermatitis, vol. 83, no. 2, pp. 174-175, 2020.

[25] C. Patruno, S. P. Nisticò, G. Fabbrocini, and M. Napolitano, "COVID-19, quarantine, and atopic dermatitis," Medical Hypotheses, vol. 143, Article ID 109852, 2020.

[26] D. Tiedemann, M. L. Clausen, S. M. John, I. Angelova-Fischer, S. Kezic, and T. Agner, "Effect of glove occlusion on the skin barrier," Contact Dermatitis, vol. 74, no. 1, pp. 2-10, 2016.

[27] P. Lin, S. Zhu, and Y. Huang, "Adverse skin reactions among healthcare workers during the coronavirus disease 2019 outbreak: a survey in Wuhan and its surrounding regions," British Journal of Dermatology, vol. 183, no. 1, pp. 190-192, 2020.

[28] G. Cavanagh and C. Wambier, "Rational hand hygiene during COVID-19 pandemic," Journal of the American Academic of Dermatology, vol. 82, no. 6, p. e211, 2020. 
[29] T. H. Mekonnen, D. G. Yenealem, and B. M. Tolosa, "Selfreport occupational-related contact dermatitis: prevalence and risk factors among healthcare workers in Gondar town, Northwest Ethiopia, 2018-a cross-sectional study," Environmental Health and Preventive Medicine, vol. 24, no. 1, p. 11, 2019.

[30] D. K. Mal and B. Mukherjee, "Educational motivation and legislative approaches for safe and effective hand washes and hand rubs in hand health care during COVID-19 Pandemic," Asia Pacific Journal of Public Health, 2020.

[31] S. J. Pidot, W. Gao, A. H. Buultjens, I. R. Monk, R. Guerillot, and G. P. Carter, "Increasing tolerance of hospital Enterococcus faecium to handwash alcohols," Science Translational Medicine, vol. 10, p. 452, 2018.

[32] T. Sandle, "Coronavirus pandemic shortages and the risks of using ineffective hand sanitisers in cleanrooms," Clean Air Journals, 2020.

[33] L. D. Moore, G. Robbins, J. Quinn, and J. W. Arbogast, "The impact of COVID-19 pandemic on hand hygiene performance in hospitals," American Journal of Infection Control, vol. 20, p. 30805, 2020.

[34] D. M. Elston, "Occupational skin disease among health care workers during the coronavirus (COVID-19) epidemic," Journal of the American Academy of Dermatology, vol. 82, no. 5, pp. 1085-1086, 2020.

[35] J. Donovan, I. Kudla, L. D. Holness, S. Skotnicki-Grant, and J. R. Nethercott, "Skin reactions following use of N95 facial masks," Dermatitis, vol. 18, no. 2, p. 104, 2007.

[36] Y. Yan, H. Chen, L. Chen, B. Cheng, P. Diao, and L. Dong, "Consensus of Chinese experts on protection of skin and mucous membrane barrier for healthcare workers fighting against coronavirus disease 2019," Dermatologic Therapy, vol. 33, no. 4, p. e13310, 2020.

[37] S. Den Boon, C. Vallenas, M. Ferri, and S. L. Norris, "Incorporating health workers' perspectives into a WHO guideline on personal protective equipment developed during an Ebola virus disease outbreak," F1000Research, vol. 7, p. 45, 2018.

[38] B. Bhoyrul, K. Lecamwasam, M. Wilkinson, F. Latheef, S. J. Stocks, and R. Agius, "A review of non-glove personal protective equipment-related occupational dermatoses reported to EPIDERM between 1993 and 2013," Contact Dermatitis, vol. 80, no. 4, pp. 217-221, 2019. 